Mujeres matemáticas: análisis del caso en México

\title{
MUJeres MATEMÁtICAS: ANÁLISIS DEL CASO EN MÉXICO
}

\author{
Rosa María González Jiménez \\ Universidad Pedagógica Nacional \\ México, D. F.
}

Todo conocimiento abstracto, todo conocimiento árido,

debe de ser dejado a la mente laboriosa y sólida del hombre (...), por ello, las mujeres nunca aprenderán geometría.

\section{Immanuel Kant}

\section{INTRODUCCIÓN}

Es un hecho conocido que las mujeres participan menos que los hombres en el área de las ciencias exactas, tanto a nivel internacional (UNESCO, 1998) como en México (Boch y Trigueros, 1996; INEGI, 2000a). Bastante menos se conoce de su incursión específicamente en el campo de las matemáticas (Hanna, 1996).

Varias investigadoras coinciden en que históricamente las matemáticas se han tipificado como un dominio masculino, situación que limita su participación como estudiantes y profesionales de las matemáticas (Burton, 1996; Parker, Rennie y Fraser, 1995; Fennema y Leder, 1990).

El propósito de este trabajo es presentar una visión amplia de la participación de mujeres matemáticas en el país. En la primera parte analizamos información a partir de que se establece como programa de formación en matemáticas en la Universidad Nacional Autónoma de México (1935), hasta la fecha, centrándonos en el porcentaje de 
mujeres que estudian y de las que ejercen docencia e investigación. En la segunda parte del texto presentamos una semblanza de diez y ocho mujeres matemáticas de primer nivel, información que obtuvimos a través de un cuestionario abierto.

Esperamos que el trabajo llene un vacío de información y oriente algunas políticas en materia de igualdad de oportunidades para las mujeres, tanto en la enseñanza de las matemáticas en los diferentes niveles educativos, como en el ejercicio profesional.

\section{Primera parte}

\section{Estudiantes de matemáticas. Antecedentes}

En 1935 por primera vez en México se ofrece un programa de formación matemáticas como licenciatura, en el Departamento de Físico-Matemáticas de la Universidad Autónoma de México (UNAM), que para 1937 adquiere el rango de Escuela dentro de la Facultad de Ciencias. De acuerdo con los datos disponibles ${ }^{1}$, en la década de los cuarenta había en promedio 27 estudiantes inscritos por año (rango 10 a 37). En el año de 1940 estudiaban matemáticas un mayor número de mujeres que de hombres (16 y 5, respectivamente), observándose a partir de 1944 una tendencia decreciente de mujeres y creciente de hombres, a partir de 1946 (gráfico 1). Entre los años de 1940 y 1948 las mujeres representaron en promedio el 34 por ciento (rango del 2 por ciento al 72 por ciento) de la matrícula en matemáticas.

\footnotetext{
${ }^{1}$ Las estadísticas históricas de la UNAM reportan datos de estudiantes de 1925 a 1955; sin embargo, no en todos los años se reportan desagregados por carrera en la Facultad de Ciencias, o por sexo en la carrera de Matemáticas.
} 


\section{Gráfico 1}

Número de alumnas y alumnos

inscritos en Matemáticas en la Facultad de Ciencias de la UNAM.

Años 1940 a 1948

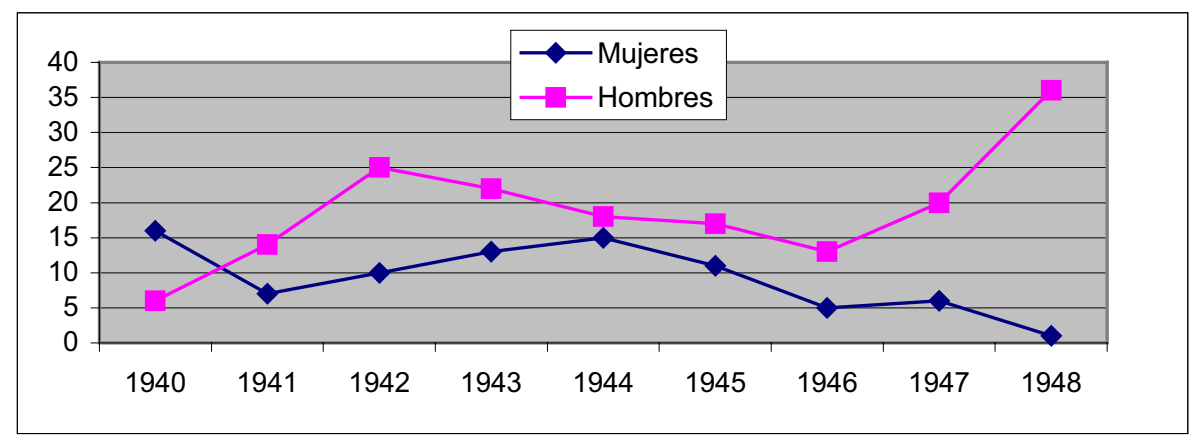

En 1937 la UNAM otorgó por primera vez un grado de licenciatura en matemáticas; tres mujeres se titularon en la década de los cuarentas: Rosa Guirre Sánchez, Carmen Alburquerque García y Ana María Flores; esta última ocupó un puesto destacado en la Secretaría de Industria y Comercio y promovió el desarrollo de la estadística. En el nivel de maestría, Enriqueta González Baz y de la Vega (1944) y María del Pilar Mercado Domenech (1947) obtuvieron el grado (Rivaud, 2000). Por su parte, Silvia de Nymet Urbina se graduó de maestría en el Instituto Politécnico Nacional (IPN) en 1964, y fue la primera doctora en matemáticas titulada en el país, grado que obtuvo en 1966 en la misma institución (Centro de Investigación Avanzada, 2001).

Para el año de 1960 había en la UNAM un total de 126 estudiantes en la carrera de matemáticas, de los cuales el 27 por ciento eran mujeres (UNAM, 1961). En 1967 hay un decremento importante de estudiantes en la carrera, registrándose sólo 24, cantidad similar a la de principios de los años cuarenta: 33 por ciento fueron mujeres (UNAM, 1968). 
Como señala Rivaud (2000), la escasa demanda por parte del alumnado fue una limitación para el desarrollo de las matemáticas en México. Para el año de 1945 del total de mujeres matriculadas en la UNAM, el 38 por ciento estaba en Matemáticas, y del total de hombres el 13 por ciento. Las carreras con mayor participación de mujeres, en ese año, eran Filosofía (18.0 por ciento), Medicina (14.8 por ciento) y Enfermería (13.6 por ciento); y de los hombres, Medicina (38.4 por ciento), Jurisprudencia (17.1 por ciento) y Administración (12.4 por ciento) (UNAM, 1959).

\section{Estudiantes de matemáticas. Época actual}

A principios de 1968 había en el país doce Facultades de Ciencias, de las cuales en siete se ofrecía la licenciatura en Matemáticas (Facultad de Ciencias de la UNAM, Escuela Superior de Física y Matemáticas del IPN, Escuela de Altos Estudios de la Universidad de Sonora, Instituto Tecnológico de Monterrey, Universidad Autónoma de Nuevo León, Universidad Veracruzana y Universidad Autónoma de Yucatán). El número de matemáticos en toda la república ascendía aproximadamente a 120 (Arvizu, 1998).

A través de los años, se han venido modificando el nombre de los programas de estudio que ofrecen las instituciones de educación superior. En la formación matemática es posible hacer una tipificación -bastante arbitraria- en tres grandes ramas: matemática básica, aplicada y estadística. Entre $1977^{2}$ y 2001 las mujeres pasan del 26.0 por ciento de la población escolar al 37.5 por ciento. Por área, en matemáticas su participación se ha mantenido reducida ( 25.3 por ciento en 1977 y 37.1 por ciento en 2001) en comparación estadística (39.1 por ciento a 44.8 por ciento, respectivamente).

\footnotetext{
${ }^{2}$ En 1977 es el primer año que la ANUIES presenta datos desagregados por sexo.
} 
Mujeres matemáticas: análisis del caso en México

Tabla 1

Porcentaje de mujeres inscritas

en una licenciatura en Matemáticas en México.

Años 1977 a 2001

\begin{tabular}{llccc}
\hline Año & Carrera & $\begin{array}{c}\text { Total de } \\
\text { estudiantes }\end{array}$ & Mujeres & $\begin{array}{c}\text { \% } \\
\text { Mujeres }\end{array}$ \\
\hline 1977 & Matemáticas & 1197 & 303 & 25.3 \\
& Estadística & 69 & 27 & 39.1 \\
& Total & 1266 & 330 & 26.0 \\
\hline 1980 & Matemático & 1154 & 376 & 32.5 \\
& Físico-Matemático & 953 & 107 & 11.2 \\
& Total & 2107 & 483 & 22.9 \\
\hline 1985 & Matemáticas & 1924 & 452 & 23.4 \\
& Estadística & 491 & 196 & 39.9 \\
& Matemáticas Aplicadas & 88 & 45 & 51.1 \\
& Matemáticas y Comput. & 607 & 207 & 34.1 \\
& Total & 3110 & 900 & 28.9 \\
\hline 1990 & Matemático & 3110 & 748 & 24.0 \\
& Estadística & 505 & 202 & 40.0 \\
& Matemáticas Aplicadas & 336 & 121 & 36.0 \\
& Matemáticas y Comput. & 1755 & 718 & 40.9 \\
& Total & 5706 & 1789 & 31.3 \\
\hline 1995 & Matemático & 1542 & 481 & 31.1 \\
& Estadística & 160 & 80 & 50.0 \\
& Matemáticas y Comput. & 2490 & 903 & 36.2 \\
& Total & 4192 & 1464 & 34.9 \\
\hline 2001 & Matemático & 3062 & 1151 & 37.5 \\
& Estadística & 321 & 144 & 44.8 \\
& Matemáticas y Comp. Ing. & 2344 & 807 & 34.4 \\
& En Matemáticas & 321 & 144 & 44.8 \\
& Total & 6048 & 2246 & 37.1 \\
\hline
\end{tabular}

Fuente: Tabla diseñada a partir de los datos de los Anuarios Estadísticos de la ANUIES, de los años respectivos

A fin de analizar las tendencias históricas del grupo de mujeres y de hombres, elegimos el caso de la UNAM. Es cierto, como se afirma (INEGI, 2000a), que las mujeres han incrementado significativamente su presencia en estudios superiores en las 
últimas décadas; ellas incrementaron su participación en 36.2 veces como estudiantes de la UNAM entre 1945 y 1999 (de 4.205 alumnas a 152.272, respectivamente). Para los hombres el incremento en estos años fue de 12.7 veces (de 13.280 alumnos a 168.727 , respectivamente); sin embargo, específicamente en la carrera de matemáticas, el incremento de mujeres solo fue de 19.2 entre 1945 y 1999 (11 alumnas y 212, respectivamente) y el de los hombres de 23.8 (17 alumnos y 406, respectivamente).

De hecho, un menor porcentaje de alumnas estudia matemáticas en 1999 (12 por ciento) que en 1945 (26 por ciento) en relación con el total de alumnas inscritas en la UNAM en esos años. Los hombres pasaron del 13 por ciento al 24 por ciento en el período (gráfico 2).

\section{Gráfico 2}

Porcentaje de mujeres y hombres inscritos en matemáticas, en relación con el total de estudiantes por sexo en la UNAM. Años 1945 y 1999

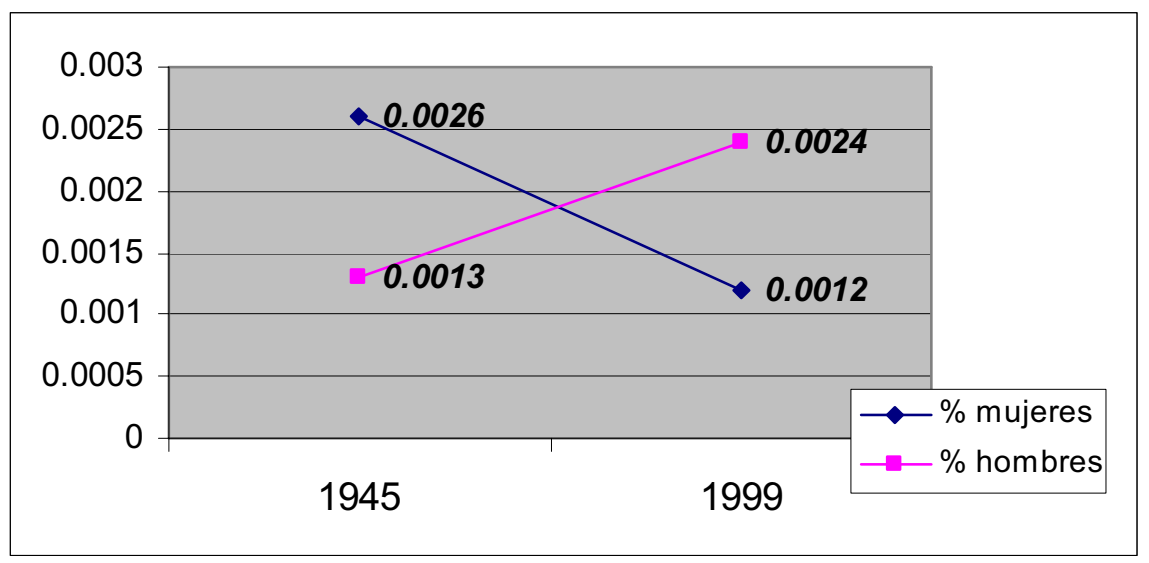

A nivel nacional, actualmente coinciden mujeres y hombres en cuanto a las tres carreras con mayor población: Contador Público (14.6 por ciento y 9.7 por ciento, respectivamente), Derecho (12.0 por ciento y 11.6 por ciento, respectivamente) y Administración (11.6 por ciento y 8.4 por ciento, respectivamente). Estos datos sugieren 
que las mujeres han incrementado significativamente su participación sólo en algunas carreras. En el estudio de las matemáticas el incremento ha sido bastante menor que el de sus compañeros.

Analizar las tendencias históricas de cara a las políticas institucionales, el prestigio de la profesión y las condiciones, intereses y decisiones de unas y otros, excede en mucho los propósitos de este trabajo.

Con los años han diversificado los programas de posgrados en matemáticas, que van desde las clásicas (básica, aplicada y estadística) hasta la matemática educativa y la ingeniería en matemáticas. Para el año de 2000, había en el país un total de 462 estudiantes en maestría, de los cuales las mujeres representaban el 31.6 por ciento. En el doctorado, de un total de 165 estudiantes el 27 por ciento son mujeres (cuadro 3) En cuanto a los estudiantes que obtienen el grado, de 47 estudiantes de maestría 27.6 por ciento son mujeres y de doctorado de 16, 18.7 por ciento (ANUIES, 2001).

\section{Cuadro 3}

Porcentaje de mujeres inscritas en un posgrado en matemáticas en México

\begin{tabular}{|c|c|c|c|}
\hline Grado/Area & $\begin{array}{c}\text { Población } \\
\text { total }\end{array}$ & Mujeres & $\begin{array}{c}\% \\
\text { Mujeres }\end{array}$ \\
\hline Maestría & 462 & 146 & 31.6 \\
\hline Ciencias matemáticas & 68 & 24 & 35.2 \\
\hline Estadística & 20 & 1 & 5.0 \\
\hline Estadística aplicada & 80 & 26 & 32.5 \\
\hline Estadística experimental & 11 & 4 & 36.3 \\
\hline Matemática educativa & 180 & 65 & 36.1 \\
\hline Matemáticas & 103 & 26 & 25.2 \\
\hline Doctorado & 165 & 46 & 27.8 \\
\hline Estadística & 16 & 3 & 18.7 \\
\hline Matemática educativa & 50 & 16 & 32.0 \\
\hline $\begin{array}{l}\text { Matemática educativa, } \\
\text { informática }\end{array}$ & 24 & 3 & 12.5 \\
\hline Matemáticas & 75 & 24 & 32.0 \\
\hline
\end{tabular}

(ANUIES, 2001) 
Sin embargo, dependiendo de la institución, hay importantes variaciones. Por ejemplo, entre los años 1961 y 2002 se han titulado en los posgrados en Matemáticas del Centro de Investigación Avanzada del IPN - una de las instituciones con alto prestigio en el campo- 203 estudiantes en maestría (11 por ciento son mujeres), y 55 de doctorado (16 por ciento son mujeres) (CINVESTAV, 2001).

\subsection{Profesoras e investigadoras en matemáticas}

En junio de 1942 inició sus actividades el Instituto de Matemáticas de la UNAM; en 1962 se crea el Centro de Estudios Avanzados del Instituto Politécnico Nacional (IPN), ambas con el propósito de desarrollar investigación; posteriormente inician actividades otras instituciones, tanto en el Distrito Federal como en los estados (Rivaud, 2000).

De las doce instituciones con programas de posgrado y/o investigación en matemáticas de las que fue posible conseguir información, siete se ubican en el Distrito Federal y el resto en algún estado de la república mexicana ${ }^{3}$. De una planta total de 591 académicos/as que laboran en estas instituciones, el 18.4 por ciento son mujeres. Al analizar por estado, la relación es de 20.9 por ciento en el Distrito Federal y el 14.2 por ciento en los estados. Los mayores porcentajes de mujeres, en relación con los hombres, se encuentran en el Departamento de Matemática Educativa del CINVESTAV (35.7 por ciento) y en el Departamento de Matemáticas de la UNAM (33.3 por ciento). Los institutos o departamentos que mantienen altos índices de investigación, de acuerdo con Rivaud (2000), como el Instituto de Matemáticas de la UNAM, el Departamento de Matemáticas del CINVESTAV, el Departamento de Matemáticas de la UAM-I y el Centro de Investigaciones Matemáticas de Guanajuato, es mucho menor la participación de mujeres (entre 8.6 por ciento y 14.7 por ciento).

\footnotetext{
${ }^{3}$ La información la recabamos de los datos que cada institución reporta en su pagina Web, las cuales ofrecen información entre 1998 a 2002, por lo que pueden no coincidir puntualmente con la información actual.
} 
Mujeres matemáticas: análisis del caso en México

\section{Tabla 4}

Porcentaje de mujeres en docencia

e investigación en Matemáticas en México

\begin{tabular}{|c|c|c|c|c|}
\hline Institución/Estado & Posgrado & $\begin{array}{c}\text { Planta } \\
\text { Académica }\end{array}$ & Mujeres & $\begin{array}{c}\% \\
\text { Mujeres }\end{array}$ \\
\hline $\begin{array}{l}\text { Instituto de Matemáticas, UNAM } \\
\text { (D.F., Morelia y Cuernavaca) }\end{array}$ & & 95 & 11 & 11.5 \\
\hline $\begin{array}{l}\text { Instituto de Inv. Matemáticas } \\
\text { Aplicadas } \\
\text { y en Sistemas UNAM (D.F.) }\end{array}$ & & 58 & 13 & 22.4 \\
\hline $\begin{array}{l}\text { Departamento de Matemáticas. } \\
\text { CINVESTAV. } \\
\text { IPN (D.F.) }\end{array}$ & $\begin{array}{c}\text { Doctorado } \\
\text { Maestría }\end{array}$ & 23 & 2 & 8.6 \\
\hline $\begin{array}{l}\text { Departamento de Matemática } \\
\text { Educativa. } \\
\text { CINVESTAV. IPN (D.F.) }\end{array}$ & $\begin{array}{c}\text { Doctorado } \\
\text { Maestría }\end{array}$ & 28 & 10 & 35.7 \\
\hline $\begin{array}{l}\text { Departamento de Matemáticas. } \\
\text { UAM-I (D.F.) }\end{array}$ & $\begin{array}{c}\text { Doctorado } \\
\text { Maestría }\end{array}$ & 61 & 9 & 14.7 \\
\hline $\begin{array}{l}\text { Centro de Investigación en } \\
\text { Matemáticas } \\
\text { (Guanajuato) }\end{array}$ & $\begin{array}{c}\text { Doctorado } \\
\text { Maestría }\end{array}$ & 65 & 8 & 12.3 \\
\hline $\begin{array}{l}\text { Instituto de Física y Matemáticas. } \\
\text { UMSNH } \\
\text { (Michoacán) }\end{array}$ & $\begin{array}{c}\text { Doctorado } \\
\text { Maestría }\end{array}$ & 14 & 1 & 7.0 \\
\hline $\begin{array}{l}\text { Facultad de Ciencias Físico } \\
\text { Matemáticas. } \\
\text { BUAP (Puebla) }\end{array}$ & $\begin{array}{c}\text { Doctorado } \\
\text { Maestría }\end{array}$ & 38 & 5 & 13.0 \\
\hline $\begin{array}{l}\text { Departamento de Matemáticas. } \\
\text { Facultad de Ciencias. UNAM } \\
\text { (D.F.) }\end{array}$ & $\begin{array}{c}\text { Doctorado } \\
\text { Maestría }\end{array}$ & 99 & 33 & 33.3 \\
\hline $\begin{array}{l}\text { Escuela Superior de Físico - } \\
\text { Matemáticas. } \\
\text { IPN (D.F.) }\end{array}$ & Maestría & 9 & 0 & 0 \\
\hline $\begin{array}{l}\text { Instituto de Econometría, } \\
\text { Estadística } \\
\text { e Informática. EP (Chapingo) }\end{array}$ & Maestría & 95 & 15 & 15.7 \\
\hline $\begin{array}{l}\text { Instituto Tecnológico de Estudios } \\
\text { Superiores } \\
\text { (Monterrey) }\end{array}$ & Maestría & 6 & 2 & 33.3 \\
\hline Total & & 591 & 109 & 18.4 \\
\hline
\end{tabular}


Fuente: Tabla diseñado con base en la información que presenta cada institución en su página $\mathrm{Web}$. El sexo lo identificamos a través del nombre del investigador/a. En los casos en que había duda (por ejemplo, Refugio, Alin), confirmamos telefónicamente el dato.

Por otra parte, en el Área de Físico-Matemáticas del Sistema Nacional de Investigadores tiene registrados 1.126 académicos, de los cuales 150 son mujeres (13.3 por ciento) (Consejo Nacional de la Mujer, 1999). La Academia Mexicana de Ciencias, institución fundada en agosto de 1959, que agrupa a la mayoría de las y los investigadores con reconocida trayectoria, en la sección de matemáticas hay 84 miembros registrados, de los cuales 6 (7.1 por ciento) son mujeres. En el siguiente gráfico, se presenta el concentrado del porcentaje de participación de mujeres en matemáticas en los diferentes niveles.

\section{Gráfico 3}

Porcentaje de mujeres estudiantes, graduadas, profesoras y/o investigadoras en Matemáticas en México

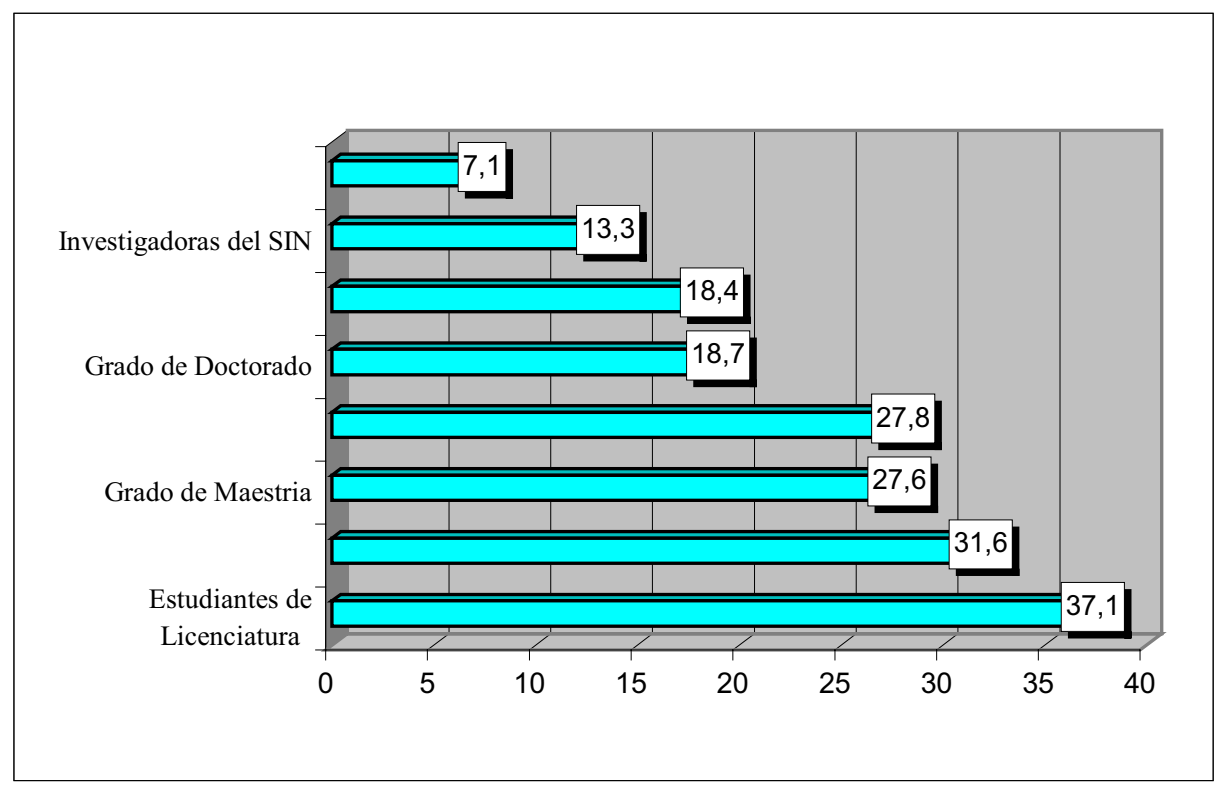


Con base en los datos es posible concluir que las mujeres, proporcionalmente, no sólo no han incrementado su participación como estudiantes de matemáticas, sino que la han disminuido, en comparación con los años cuarenta. El área en que menos participan es matemática básica. También, en menor proporción que los hombres, llegan a titularse.

Como profesoras e investigadoras representan un porcentaje reducido, reduciéndose aún más en la medida en que la institución cuenta con mayor prestigio en investigación o se ubica fuera del Distrito Federal. Por área de especialidad, las investigadoras están en mayor proporción en matemática educativa y estadística que en matemática básica o aplicada.

\section{SEGUNDA PARTE}

\section{Semblanza de matemáticas mexicanas}

"Yo soy de la generación que ha tenido que abrir brecha, demostrar que las mujeres también podemos hacer matemáticas de muy buen nivel”

"Como dicen, al hombre se le cree el doctorado y la mujer lo tiene que demostrar"

Matemáticas mexicanas

Los trabajos que recuperan a mujeres matemáticas en la historia relatan que aunque muy pocas tuvieron la oportunidad de cursar estudios formales, no por ello dejaron de hacer importantes aportaciones a la disciplina. Las historias de Hypatía, María Gaëtana Agnesi, Sophie Germaín o Ada Lovelance son algunos ejemplos de matemáticas notables que en general enfrentaron grandes obstáculos para desarrollar y 
dar a conocer su trabajo. Frecuentemente sus escritos se publicaron a nombre de su esposo, hermano o colega (Alic, 1991; Yount, 1999).

En México está por escribirse la historia de las aportaciones que han hecho las mujeres a la disciplina, así como aspectos culturales y políticas institucionales que han favorecido y limitado su participación. Localizamos sólo el escrito que relata la vida y aportes de una reconocida matemática mexicana: Graciela Salicrup, quien trabajó en el Instituto de Matemáticas de la UNAM, desarrollando importantes aportes al campo de la Topología. Graciela fallece prematuramente, en un accidente ocurrido en su centro de trabajo, el 28 de julio de 1982 (Galeana, 1990).

En este apartado describimos algunas características y opiniones de mujeres matemáticas. Diseñamos un cuestionario abierto que interroga acerca de la trayectoria personal y profesional, el cual enviamos por Internet al correo electrónico de 45 mujeres que desarrollan investigación de primer nivel. De éstas respondieron 18, de las cuales el 17 tiene el grado de doctorado y una de maestría. El 61.1 por ciento cursó sus estudios de posgrado en el extranjero y el resto en México. Ellas laboran en las principales instituciones que realiza investigación matemática en el país: Instituto de Matemáticas e Instituto de Investigación en Matemáticas Aplicadas, Sistemas y Servicios (UNAM); Departamento de Matemáticas y Departamento de Matemática Educativa (IPN); Centro de Investigación Matemática de Guanajuato y Departamento de Matemáticas (UAM- I).

Los datos que a continuación se reportan no pretenden generalizarse para todas las matemáticas del país, aunque no dejan de ser un indicador de sus condiciones. Realizamos diversos cruces entre variables (edad, estado civil, número de hijos, escolaridad del padre y la madre, lugar de nacimiento, lugar de estudios de posgrado, etcétera) utilizando la prueba no paramétrica U de Mann-Whitney; se reportan solo aquellos casos que fueron significativos en un nivel de $\mathrm{p}<.05$.

\subsection{Aspectos sociodemográficos}

La edad promedio de las mujeres que respondieron es de 45.8 (rango 33 a 58 
años), el 77.8 por ciento nace en el Distrito Federal y el resto en otros estados de la república mexicana. En promedio tienen 1.4 hijos (rango 0 a 3). Dos factores influyen en la tasa de fecundidad de las mujeres: el nivel escolar y la actividad productiva; existe una relación inversa entre el nivel escolar y el número de descendientes, así como entre si las mujeres desarrollan una actividad productiva y el número de hijos. De acuerdo con los datos del Instituto Nacional de Estadística, Geografía e Informática (2000b), la tasa global de fecundidad de las mujeres económicamente activas es de 2.0 contra 3.4 de las no activas. En cuanto a la escolaridad, las mujeres con secundaria o más presentan una tasa de fecundidad del 2.2 contra el 4.7 de mujeres sin instrucción. Por la cantidad de hijos/as, los datos de las matemáticas es probable que sean similares a la de otras mujeres profesionistas con alta escolaridad.

Por su estado civil, el 55.6 por ciento están casadas, el 5.6 por ciento son separadas o divorciadas, el 22.2 por ciento son solteras y 16.7 por ciento vive en unión libre. A nivel nacional el 12.1 por ciento de las mujeres de 12 años o más son solteras o vive en unión libre (INEGI, 2000b). En el caso de las maestras de primaria, el 17 por ciento son solteras y el 3 por ciento declaran unión libre (De Ibarrola, Silva y Castrejón, 1997). No existe información al respecto de mujeres que ejercen otras profesiones, aunque se sabe que la conjunción de cargos de poder (material y/o simbólico), alta escolaridad e independencia económica favorece su decisión de no establecer contratos matrimoniales, lo que no implica necesariamente ausencia de pareja. Por su parte, el porcentaje de divorcios es menor comparado con mujeres que ejercen otras profesiones, que es del 22.8 por ciento (INEGI, 2000b).

La escolaridad promedio de su padre es de bachillerato (rango doctorado a primaria incompleta) y de su madre secundaria (rango licenciatura a primaria incompleta). Un alto porcentaje (72.2 por ciento) cursó la educación básica en escuelas particulares y el resto en públicas. En la década de los setenta el promedio escolar de la población a nivel nacional ascendía a tercero de primaria (INEGI, 2000b), por lo que se infiere un alto nivel escolar familiar. 
Encontramos diferencias significativas por el nivel de estudios del padre. Los padres con más alto nivel escolar, en mayor medida matricularon a sus hijas en escuelas privadas que los padres con menor nivel escolar, quienes más frecuentemente las inscribieron en escuelas públicas ( $\mathrm{U} 16.500, \mathrm{p}=.044)$.

Es conocido el desprestigio que tiene la educación básica oficial entre estratos medios y altos, quien prefiere enviar a sus hijos/as a escuelas particulares. Estudiantes que, a la postre, en promedio ocupan las posiciones más prestigiadas social y económicamente.

Por otra parte, algunas investigaciones han encontrado que las escuelas unisexuales favorecen que las chicas decidan estudiar matemáticas (Haag, 2000; Mael, 1998). No identificamos evidencias al respecto, ya que sólo dos de las matemáticas estudiaron su educación básica y/o media en escuelas exclusivas de alumnas.

\subsection{Antecedentes escolares}

En general refieren buenos recuerdos de lo que fue su educación básica (46.8 por ciento excelente y 36.5 por ciento buena) y la gran mayoría (76.5 por ciento) obtuvo altas calificaciones en matemáticas en primaria y secundaria. Casi todas (88.9 por ciento) cursaron sus estudios de licenciatura en una institución pública y dos de ellas su licenciatura de origen es en el área de ciencias sociales. Contrario a lo que sucede en educación básica, en el país la educación superior pública cuenta con mayor prestigio que la privada, en especial en áreas científicas.

El 72.2 por ciento de ellas, al estar cursando el bachillerato ya habían decidido estudiar matemáticas o una carrera vinculada con ésta.

\subsection{Razones para estudiar matemáticas}

Como primera opción, el 68 por ciento manifiestan que fue el gusto o placer que les generaba resolver problemas matemáticos el aspecto que más influyó en su 
decisión ("tengo una razón principal: era lo que más me gustaba").

Como segunda opción refieren su rechazo a disciplinas poco exactas u objetivas ("Las carreras sociales me parecía que dependían de una corriente y que no era posible determinar exactamente qué pasaba"; "Las disciplinas sociales me parecían poco serias") o por el tipo de pensamiento que involucra su estudio ("no hay que memorizar nada, sino que aprender a pensar y razonar").

En tercer lugar refieren la influencia de profesores/as ("Me empezaron a interesar las matemáticas en $2^{\circ}$ de secundaria. Me gustaba especialmente el rigor de Sarita López de Llergo"), influencia familiar ("Mi papá, al ver nuestras calificaciones, veía que no hubiera notas rojas y luego sólo se fijaba en la de matemáticas"), la belleza de la disciplina y lo barato que resulta la formación ("Es una carrera relativamente barata, porque originalmente quería estudiar arquitectura").

\subsection{Discriminación educativa y laboral}

Muy pocas (16.7 por ciento) refieren alguna forma de discriminación por el hecho de ser mujeres en su formación; aquellas que responden afirmativamente comentan

"Los profesores en los Estados Unidos me veían con condescendencia por ser mujer y del tercer mundo".

"Cuando pedí complemento de beca de doctorado, como casada, me dijeron que únicamente me la darían si mi esposo estaba discapacitado. Claramente a los hombres se les otorgaba sin hacer este tipo de preguntas".

El 50 por ciento no recuerda haber tenido una mujer como profesora en sus estudios de posgrado, y el 23.5 por ciento recuerda sólo una. Aquellas que mencionan que sí tuvieron profesoras, son del área de ciencias sociales o en mayor medida su especialidad es estadística. 
En cuanto a discriminación laboral, el 33.3 por ciento señala alguna forma de discriminación. Manifiestan que no es un hecho conciente por parte de sus colegas, sino un problema del sistema social. Entre las cuestiones que refieren con mayor frecuencia esta la "doble jornada" de trabajo. Es sabido que las mujeres dedican más del doble de tiempo en el trabajo doméstico y el cuidado de los hijos/as que los hombres (INEGI, 2000b); también señalan el hecho de que socialmente a los hombres se les considera más adecuados para ocupar cargos y responsabilidades. A continuación se describen algunos de sus comentarios al respecto

"Tengo que escribir un cierto número de artículos al año; mis colegas, los que cuentan con infraestructura "femenina", sufren menos por falta de tiempo para cumplir con su trabajo; también tienen menos posibilidades de sufrir de tensión y cansancio constantes, enemigos mortales de la producción intelectual."

"Hay una discriminación sutil, no abierta. Para promociones o distinciones se considera naturalmente al hombre y la mujer lo tiene que pedir".

"Durante el primer trabajo y antes del posgrado (...) menor salario, menos oportunidades de crecimiento, frases como, 'dado que eres mujer y trabajas para ayudar a tu esposo', etc. etc., y otras concepciones erróneas acerca del quehacer profesional de una mujer".

"No pude ingresar como candidato al Sistema Nacional de Investigadores cuando todavía tenía la edad adecuada porque mi hija era muy pequeña y no podía darme a basto para cuidarla y producir artículos; ahora tampoco puedo porque no he acumulado la suficiente cantidad de artículos".

\subsection{Desarrollo personal y profesional}

Se les interrogó si consideraban que su desarrollo profesional había limitado su vida personal. La pregunta se formuló en estos términos, ya que en todos los casos son mujeres reconocidas profesionalmente. Varias de ellas señalaron que la pregunta debió haberse formulado al revés, si su vida personal limitó su desarrollo profesional. El 47.1 
Mujeres matemáticas: análisis del caso en México

por ciento respondió en este sentido, y el 23 por ciento no respondió esta pregunta.

Entre las que respondieron negativamente, comentan

"Al contrario, creo que el hecho de apasionarse por una actividad enriquece las relaciones con otras personas y en particular la relación de pareja".

"Un factor importante es que ambos tenemos la misma profesión y el mismo grado, a diferencia de otros casos".

De las que respondieron afirmativamente a la pregunta, señalan

"Esta pregunta es difícil de contestar (...). Cuando estoy inspiradísima, escribiendo un artículo, y me llaman de la escuela para decirme que mi hija tiene calentura, tengo que interrumpir mi trabajo para ir a recogerla; la verdad, en esos momentos, me gana la frustración (...); pero en otras ocasiones, cuando no tengo inspiración y no me dan ganas ni de poner el pie en el instituto, quisiera tomar a mi hija e irme "de pinta" a Chapultepec con ella (...). Tengo pocos momentos para compartir diversiones con ella".

"Las matemáticas en México están muy idealizadas; creen que uno es un dios inalcanzable, por lo que tener pareja fuera del ambiente es complicadísimo".

"Para las que somos madres resulta muy complicado cumplir con los requisitos del sistema y desgraciadamente la edad reproductiva coincide con la época en que aún no se suele tener la definitividad".

Cuando menos tres hipótesis sugieren las respuestas, que no son excluyentes entre sí: 1) Valoran su desarrollo profesional y consideran que éste ha enriquecido sus relaciones personales; 2) Algunas matemáticas consideran que hubieran podido desarrollarse más profesionalmente, si no fueran las principales responsables de los hijos/as. 3) Hay alguna resistencia para reconocer que su vida profesional pudo haber constituido una limitación en su vida personal.

Como bien lo señala una de ellas

"En realidad, uno de los secretos de aprender a vivir consiste en encontrar el equilibrio entre lo que una hace para ganarse el pan, y lo que hace para ser feliz". 


\subsection{Matemáticas mexicanas notables}

A la pregunta de que si en México hay o hubo matemáticas que no hayan recibido el reconocimiento que merecían, el 65 por ciento manifiestan no tener información y el 12 por ciento respondió negativamente. De aquéllas que contestaron afirmativamente, mencionan a Graciela Salicurp, Monica Clapp, Emilia Caballero y Sarita López de Llergo.

\section{CONCLUSIONES Y DISCUSIÓN}

En el país, como en otras partes del mundo, hacer matemáticas es una actividad tipificada como un dominio propio para varones. Aunque en la última década las jóvenes han incrementado su participación en la disciplina, conforme se asciende de nivel, ellas disminuyen su presencia en el campo.

Las mujeres matemáticas que ejercen la investigación y la docencia en instituciones de primer nivel, en general provienen de familias con alta escolaridad, cursaron su educación básica mayoritariamente en escuelas privadas, consideran haber tenido buenos/as profesores/as de matemáticas y obtuvieron buenas calificaciones en la materia.

Mejorar la enseñanza de las matemáticas en educación básica, a través de la actualización magisterial, es un aspecto crítico para incrementar la cantidad de las y los estudiantes que decidan estudiar la disciplina, incorporando estrategias educativas que se han probado para que más niñas se interesen por las matemáticas (González et alii, 2001; Sanders, 1994).

La licenciatura la estudiaron mayoritariamente en una institución pública, y aquéllas cuyos padres tienen mayor escolaridad, con más frecuencia estudiaron el posgrado en el extranjero.

En su vida personal identificamos diferencias con sus colegas varones. Los datos sugieren una tendencia mayor de las matemáticas a permanecer solteras o en 
unión libre que el resto de la población; el dato es interesante ya que esta tendencia se refiere también para las primeras profesionistas mexicanas de finales del siglo XIX (González y Toriz, 2000) y principios del XX (Cano, 1999). En ese tiempo a las mujeres inteligentes se consideraban una "singularidad" que producía temor.

Algunas de ellas refieren discriminación laboral, principalmente por la doble jornada de trabajo y falta de reconocimiento profesional. Otra diferencia con sus colegas varones es la tensión que genera la disyuntiva de desarrollar los trabajos necesarios para cumplir las metas de producción y el bienestar de los hijos/as.

En mi experiencia como psicoanalista, aquellas mujeres que ocupan cargos de poder, invariablemente refieren sentirse culpables por la salud, comportamiento o rendimiento escolar de sus hijos/as. Jamás he escuchado a un hombre responsabilizarse en este sentido por el tiempo que dedican a su trabajo.

Por otra parte, si bien el Sistema Nacional de Investigadores (SNI) concede un año de plazo para las investigadoras que acaban de parir (Consejo Nacional de la Mujer, 1999), el proceso de evaluación para acceder o permanecer en el SNI es probable que tenga sesgos sexistas. En especial en campos tipificados como un dominio masculino se han identificado sesgos importantes en contra de las mujeres en la forma como se evalúa su producción (Keller, 1991). En todo caso queda pendiente como una interesante pregunta de investigación.

Además, es importante promover entre las investigadoras que concursen para ingresar en el SNI, ya que proporcionalmente deciden participar menos que los hombres en el proceso de evaluación, lo que sugiere autocensura de su parte (Consejo Nacional de la Mujer, 1999).

Dos cuestiones destacan como razón para haber estudiado matemáticas, el gusto por la disciplina y su rechazo al conocimiento ambiguo o subjetivo; en este punto difieren de mujeres que estudian ciencias sociales y humanas, quienes suelen argumentar intereses sociales y humanitarios, que sería interesante profundizar en futuras investigaciones. 
En tanto se continúe enseñando a niñas y mujeres que su función principal es el cuidado de los otros, y las matemáticas se consideren un conocimiento abstracto, con escaso vínculo con la vida social, más difícilmente ellas optarán por estudios superiores vinculados con la disciplina. 
Mujeres matemáticas: análisis del caso en México

\section{BIBLIOGRAFÍA}

ALIC, M. (1991): El legado de Hypatia. Historia de las mujeres en la ciencia desde la antigüedad hasta fines del siglo XIX. México: Siglo XXI Editores.

Asociación Nacional de Universidades e Instituciones de Educación Superior (1977, 1980, 1985, 1990, 1995, 2001): Anuarios estadísticos. Población escolar de licenciatura en universidades e institutos tecnológicos. México: Asociación Nacional de Universidades e Instituciones de Educación Superior.

- (2001): Anuario estadístico. Población escolar de posgrado. México: Asociación Nacional de Universidades e Instituciones de Educación Superior.

Boch, C. y Trigueros, M. (1996): "Gender and mathematics in México". En G. HANNA (ed.): Towards gender equity in mathematics education. An ICMI study. The Netherlands: Klower Academic Publishers.

BURTON, L. (1996): Gender and mathematics. An international perspective. London: CASSEL.

CANO, G. (1999): La soltería y el desarrollo intelectual de las mujeres: ¿un matrimonio bien avenido? Cuidado con el corazón. México: Instituto Nacional de Antropología e Historia.

Consejo Nacional de la Mujer (1999): El enfoque de género en la producción de las estadísticas educativas de México. México: UNIFEM/Consejo Nacional de la Mujer.

FenNemA, E. y LeDER, G.C. (1990): Mathematics and gender. New York: Teachers Collage Press.

Galeana, P. (1990): Antología de mujeres universitarias. México: Universidad Nacional Autónoma de México.

GonzÁlez, R. M.; Miguez, M. P.; Toriz, A. y PArga, L. (2001): "Estrategias educativas para la igualdad de oportunidades de alumnas y alumnos en la escuela básica y media superior”. En Entre Maestros Vol. Núm. 4, pp. 47-63. 
GONZÁLEZ, R.M. y TORIZ, A. (2000): "Primeras profesionistas mexicanas: las ventajas de la anarquía”. En Antropología No. 58, pp. 2-13.

HAAG, P. (2000): "K-12 single-sex education: What does the research say?". Documento ERIC No. ED 444758.

HanNA, G. (1996): Towards gender equity in mathematics education. An ICMI Study. The Netherlands: Kluewr Academia Publishers.

Ibarrola, M, de; Silva, G. y CASTElÁN, A. (1997): ¿Quiénes son nuestros profesores? México: Fundación SNTE para la Cultura de los Maestros.

Instituto Nacional de Geografía, Estadística e Informática (2000a): Estadísticas educativas de hombres y mujeres. México: Instituto Nacional de Estadística, Geografía e Informática.

-(2000b): Hombres y mujeres en México. México: Instituto Nacional de Estadística, Geografía e Informática / Consejo Nacional de la Mujer.

KELLER, E.F. (1991): Reflexiones sobre género y ciencia. Valencia: Edicions Alfons El Magnànim.

MAEL, F.A. (1998): "Single-sex and coeducational schooling: relationships to socioemotional and academic development". En Review of Educational Research Vol. 68, Núm. 2, pp. 101-129.

PARKER, L.H.; Rennie, L.J. y Fraser, B.J. (1995): Gender, science and mathematics. Shortening the shadow. Netherlands: Kluwer Academic Publishers.

Rivaud, J.J. (2000): “Las matemáticas. Antecedentes”. En A. MENCHACA (coord.): Las ciencias exactas en México. México: Fondo de Estudios e Investigaciones Ricardo J. Zevada / Consejo Nacional para la Cultura y las Artes/Fondo de Cultura económica.

SANDERS, J. (1994): Lifting the barriers. 600 tested strategies that really work to increase girl's participation in science, mathematics and computers. New York: Jo Sanders Publications.

UNESCO (1998): Informe Mundial sobre la Ciencia. Madrid: Santillana. 
Mujeres matemáticas: análisis del caso en México

Universidad Nacional Autónoma de México (1959, 1961, 1968, 1999): Anuario Estadístico. México: UNAM.

Yount, L. (1999): A to $Z$ of women in science and math. A biographical dictionary. New York: Facts On File Library of Word History.

\section{SITIOS WEB CONSULTADOS}

Academia Mexicana de Ciencias www.amc.unam.mx/coordinadores_seccion

ANUIES. Las diez carreras más pobladas. www.anuies.mx/estadis/licen977

Centro de Investigación Avanzada. IPN. Departamento de Matemáticas. www.math.cinvestav.mx/profes/

Arvizu, M.A. (1998): Concursos de física y matemáticas en busca de talento y vocación. http://www.iscson.uson.mx/larevista/concursos.htm

Centro de Investigación Avanzada. IPN. Departamento de Matemática Educativa. (2001). www.matedu.cinvestav.mx/

Escuela Superior de Física y Matemáticas. Maestría en Matemáticas. IPN.

www.esfm.ipn.mx/PosgradoMat.html

Facultad de Ciencias. Departamento de Matemáticas. UNAM www.fciencias.unam.mx/Docencia/Posgrado/Matematicas/

Instituto de Econometría, Estadística e Informática. Escuela de Posgraduados de Chapingo www.infocbi.uam.mx/departamentos/matematicas/profprob

Instituto de Investigaciones en Matemáticas Aplicadas, Sistemas y Servicios. UNAM www.iimas.unam.mx/

Instituto de Matemáticas. UNAM www.matem.unam.mx/investigacion

Instituto Tecnológico de Estudios Superiores. Campus Monterrey

www.mty.itesm.mx/decic/maestrias/ea/frame_claustro

Universidad Autónoma Metropolitana-Iztapalapa. Departamento de Matemáticas. www.iztapalapa.uam.mx/iztapala.www/division.cbi/matematicas/proftopo 
